\title{
Etude ethnobotanique des sous-variétés de Syzygium guineense (Will.) DC. var. macrocarpum (Engl.) F. White dans les Hautes Savanes Guinéennes (Adamaoua, Cameroun)
}

\author{
Georges Maxime LAMY LAMY*, Adamou IBRAHIMA, Dieudonné NDJONKA et Pierre \\ Marie MAPONGMETSEM
}

\author{
Département des Sciences Biologiques, Faculté des Sciences, Université de Ngaoundéré, B.P. 454 \\ Ngaoundéré, Cameroun. \\ *Auteur correspondant; E-mail: geomaxlamy@gmail.com; Tél: + 237699021552
}

\section{RESUME}

Syzygium guineense var. macrocarpum est une plante à usages multiples, à plusieurs sous-variétés et qui est menacée de disparition. Cette étude envisage de déterminer le savoir endogène, de décrire et d'évaluer les sous-variétés de la plante dans les Hautes Savanes Guinéennes (Adamaoua, Cameroun). Les méthodes ethnobotaniques dont la valeur d'usage ethnobotanique (VUE) ont été utilisées sur 195 hommes et 23 femmes. Les résultats ont montré de nombreux usages faits des parties de la plante. Les fruits faisaient l'unanimité en consommation humaine. L'huile issue des graines des fruits était comestible et commercialisée. Le savoir locale dépendait de l'âge, du genre et du groupe ethnolinguistique. La VUE a permis de classer les quatre sous-variétés de la plante, distinguables par leurs fruits en maturité complète en fonction des préférences. L'existence des sousvariétés indique une variabilité morphologique de Syzygium guineense var. macrocarpum dans la zone d'étude. Des études moléculaires pourront élucider cette variabilité. Des études de toxicités de l'huile issue des graines de la plante permettront sa valorisation.

(C) 2018 International Formulae Group. All rights reserved.

Mots clés: Ressources génétiques forestières, érosion génétique, sécurité alimentaire, connaissance traditionnelle, Cameroun.

\section{Ethnobotanical study of Syzygium guineense (Will.) DC. var. macrocarpum (Engl.) F. White sub-varieties from the Guinean Savannah Highlands (Adamawa, Cameroon)}

\begin{abstract}
The multipurpose tree Syzygium guineense var. macrocarpum has sub-varieties but it is at risk of disappearing. The study aimed at determining indigenous knowledge, describing and evaluating the sub-varieties of the plant from Guinean Savannah Highlands (Adamawa, Cameroon). The ethnobotanical methods as the ethno-botanic use value (UV) were used to interview 195 men and 23 women. The results showed many uses of the plant. The fruits were consumed by all the indigenous. By product seed oil from these fruits was commercialized and eaten. The indigenous knowledge was depended on age, gender and ethnolinguistic group. The four plant sub-varieties were classified using the UV depending on the preferences, discrimination among these sub-varieties were done by mature fruits characteristics. Due to existence of sub-varieties, Syzygium
\end{abstract}


guineense var. macrocarpum have the morphological variability in the study area. The molecular studies are required to elucidate this variability. Studies of toxicities of the plant seed oil will make it possible to value it. (C) 2018 International Formulae Group. All rights reserved.

Keywords: Forest genetic resources, genetic erosion, food security, indigenous knowledge, Cameroon.

\section{INTRODUCTION}

L'espèce Syzygium guineense (Will.) DC. var. macrocarpum (Engl.) F. White est largement distribuée en Afrique tropicale (Maroyi, 2008 ; Kimpouni, 2009). Au Cameroun, cette plante de la famille des Myrtacées est endémique des Hautes Savanes Guinéennes (Loura et al. 2000). Dans cette zone agro-écologique, de nombreuses études ont été faites sur la plante hormis celles ethnobotaniques. C'est le cas en agroforesterie (Mapongmetsem et Laissou, 2010; Doumara et Mapongmetsem, 2013), en apiculture (Tchuenguem et al., 2009), en biochimie (Loura et al., 2000) et en écologie (Ibrahima et $a l ., 2008)$. Sur le plan de la commercialisation des fruits de la plante, une quantité de $1286 \mathrm{Kg}$ de ceux-ci a été estimée à 100.000 FCFA, environ 182 dollars US (Mapongmetsem et al., 2009). Ces fruits encore à l'état sauvage ont révélé une teneur en nutriments supérieur à ceux de nombreux fruitiers déjà domestiqués (Loura et al., 2000 ; Mapongmetsem et al., 2012). Des enquêtes menées auprès des vendeuses des produits forestiers non ligneux (PFNL) des marchés périodiques des Hautes Savanes Guinéennes révèlent que la plante compte plusieurs sous-variétés. L'existence des sous-variétés de la plante a récemment été rapportée (Zigelski, 2016). Cette variabilité au sein de Syzygium guineense var macrocarpum présenterait de nombreux atouts. Malgré les usages multiples de la plante dans cette zone agro-écologique, celle-ci est classée dans la catégorie espèces menacées d'extinction en Côte d'Ivoire (Ambé et al., 2015). Au Burkina Faso, elle se raréfie (Nikema et al., 2001). Nonobstant sa diminution progressive, la plante peut être conservée dans les Hautes Savanes Guinéennes (Adamaoua, Cameroun). La formulation de cette hypothèse nous amène à faire un état du savoir endogène sur la ressource dans cette zone agro-écologique.
Ainsi, le manque d'informations sur les études ethnobotaniques relatives à la plante sera comblé. L'objectif de cette étude visait à recenser les principaux usages de $S$. guineense var macrocarpum, à décrire et à évaluer les sous-variétés de la plante. Ces informations seront utiles pour les développements futurs de programmes de protection et de conservation de l'espèce dans les Hautes Savanes Guinéennes.

\section{MATERIEL ET METHODES Sites d'étude}

Les Hautes Savanes Guinéennes de l'Adamaoua correspondent à la zone de transition entre le Nord soudano-sahélien et le Sud forestier du Cameroun. Cette zone est située entre les $6^{\text {ème }}$ et $8^{\text {ème }}$ degrés de latitude Nord et entre les $10^{\text {ème }}$ et $16^{\text {ème }}$ degrés de longitude Est. Elle compte cinq Départements à savoir : Djerem, Faro et Deo, Mayo Banyo, Mbéré et Vina. Le département de la Vina comprend huit Arrondissements (Figure 1) dont Bélel, Martap, Mbé, Ngaoundéré I, Ngaoundéré II, Ngaoundéré III, Ngang-Ha et Nyambaka. Pour ce qui est du climat, il est de type soudano-guinéen d'altitude avec 02 saisons: une saison de pluies allant d'avril à octobre et une saison sèche de novembre à mars. La pluviométrie annuelle de 1600 à 1800 $(\mathrm{mm})$ est repartie sur 7 à 8 mois (Deffo et al., 2009). Quant au sol, il se compose en majorité des structures ferralitiques rouges développées sur des vieux basaltes. Divers types de végétations y sont aussi observés. Elles vont des prairies aux savanes arbustives jusqu'aux savanes arborées dominées par Daniellia oliveri et Lophira lanceolata. La densité de ces espèces caractéristiques est en nette diminution à cause de l'action anthropique (Mapongmetsem et al., 2012). Concernant les populations, elles sont composées de plusieurs groupes ethnolinguistiques d'inégale 
répartition dont les plus représentatifs sont: Dii ou Dourou, Gbaya, Haoussa, Mbororo, Mboum et Peulh (Mapongmetsem et al., 2009; Tchobsala et Mbolo, 2013).

\section{Méthode}

L'espèce Syzygium guineense var. macrocarpum étant identifiée a l'Herbier National du Cameroun au numéro « 49885 $H N C »$ (Tankeu et al., 2016) puis répertoriée dans le site www.theplantlist.org (WCSP, 2018), nous avons entamé l'étude par un test du questionnaire sur la plante. Durant l'enquête ethnobotanique précédée $\mathrm{du}$ test $\mathrm{du}$ questionnaire auprès des femmes des marchés périodiques des Hautes Savanes Guinéennes (Adamaoua, Cameroun), il a été constaté que ces dernières s'occupaient de la cueillette, du ramassage et de la commercialisation des fruits. Elles ont permis de localiser les villages où se trouvent au moins un pied du fruitier. Au total, 218 personnes soit 195 hommes et 23 femmes âgées d'au moins 15 ans, appartenant à 15 ethnies différentes (Dii, Gbaya, Guiziga, Haoussa, Kanouri, Kany, Kobéra, Laka, Mbéré, Mboum, Ngambaye, Panah, Peulh...) ont été enquêtées. Cette limitation d'âge tient $\mathrm{du}$ fait que pour avoir une meilleure connaissance des PFNL, il faut disposer d'une certaine maturité physique et culturelle (Lougbegnon et al., 2011). Les grandes lignes du questionnaire ont été relatives aux noms en langues locales (Fulfuldé, Gbaya, etc.) de Syzygium guineense var. macrocarpum, parties ou organes de l'espèce végétale utilisés, saveur des fruits, différentes sous-variétés et leurs caractéristiques et importance de l'utilisation de la plante par catégories d'usages (alimentaire, médicinale, commerciale, bois énergie et autres). Les interviews semistructurées se sont faites au moyen des questions ouvertes, indirectes et directes auprès des hommes et des femmes séparément et en groupe de volontaires soit 6 mixtes (hommes et femmes) et 4 groupes homogènes (hommes ou femmes) en langue locale Fulfuldé. Dans chaque localité, notre présence était connue des autorités traditionnelles qui, nous proposaient un guide à chaque fois. Pour une reproductibilité des résultats, les enquêtes se sont effectuées durant trois saisons de fructification (avant, pendant et après). Cellesci ont été réparties comme suit: saison I (novembre-décembre 2014 et janvier-février 2015), saison II (avril, mai et juin 2016) et saison III (août et septembre 2017).

\section{Traitement des données \\ Calcul des valeurs d'usage ethnobotanique}

Les données collectées ont d'abord été regroupées par catégorie d'usage de chaque sous-variété au moyen d'un score d'utilisation attribué par les répondants selon chaque catégorie d'usage. La grille d'appréciation utilisée (Lougbegnon et al., 2011 ) était : $3=$ sous-variété fortement utilisée; $\mathbf{2}=$ sousvariété moyennement utilisée ; $\mathbf{1}$ = sous-variété faiblement utilisée; $\mathbf{0}=$ sous-variété non utilisée.

La valeur d'usage ethnobotanique unitaire (VUEU) et la valeur d'usage ethnobotanique totale (VUET) ont été calculées pour chaque sous-variété suivant la méthode de calcul utilisée par plusieurs auteurs (Belem et al., 2008 ; Lougbegnon et al., 2011). La valeur d'usage ethnobotanique unitaire (VUEU) d'une sous-variété au sein d'une catégorie d'usage est représentée par son score moyen d'utilisation au sein de la catégorie d'usage. La valeur d'usage ethnobotanique totale (VUET), quant à elle est calculée par la somme des valeurs d'usage d'une sous-variété au sein des différentes catégories d'usage. Leurs intérêts résident dans le fait qu'elles permettent de déterminer de façon significative les sous-variétés ayant une grande valeur d'utilisation et qu'il faudra considérer dans les futurs programmes de sélection et de domestication.

VUEU $=\sum$ Su $/ \mathbf{n}:$ avec $:$ VUEU $=$ valeur d'usage de la sous-variété unitaire pour une catégorie donnée; $\mathbf{S u}$ : score d'utilisation attribué par les répondants; $\mathbf{n}$ : nombre de réponses positives (oui) pour une sous-variété dans une catégorie d'usage donnée.

VUET $=\sum$ VUEU avec: VUET : valeur d'usage ethnobotanique total de l'espèce u, VUEU = valeur d'usage ethnobotanique d'une sous-variété donnée pour une catégorie d'usage. 


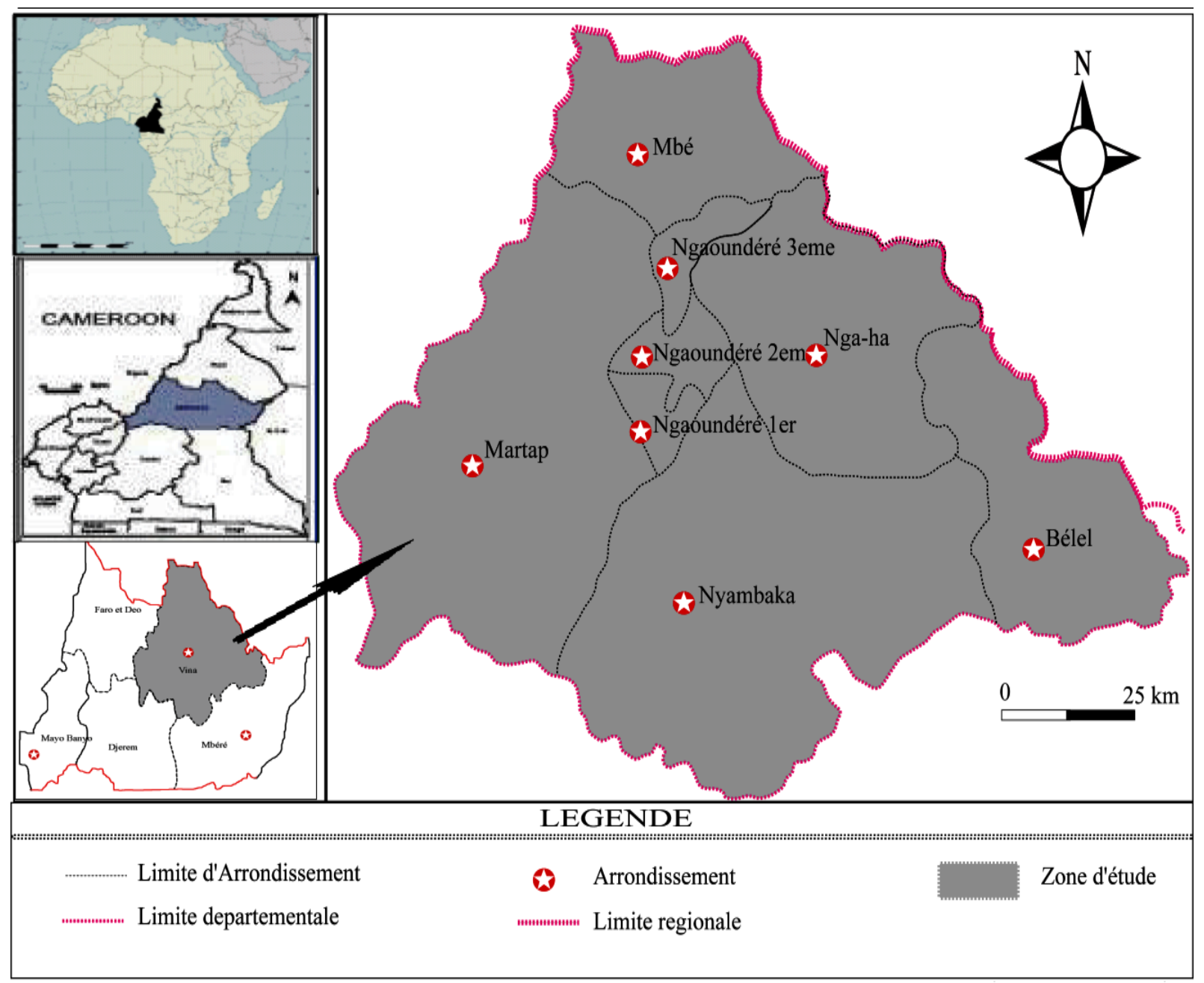

Figure 1: Localisation des 08 arrondissements ayant servi de sites d'étude dans les Hautes Savanes Guinéennes (Adamaoua, Cameroun).

\section{RESULTATS}

Savoirs endogènes et noms locaux de $S$. guineense var. macrocarpum dans les Hautes Savanes Guinéenne

Les enquêtés ont été regroupés en six groupes ethnolinguistiques suite aux similarités entre les groupes (Tableau 1). Plusieurs noms locaux ont été recensés, seules deux communautés locales désignaient la plante différemment. En effet, les Mboum $(40,37 \%)$ distinguaient quatre sous-variétés distinctes de la plante tandis que les Peulh $(35,32 \%)$ rapportaient quatre synonymes pour la nommer. Les adultes $(90,83 \%)$ avaient plus de connaissances sur la ressource végétale comparés aux jeunes $(9,17 \%)$. Pour les utilisations, celles reconnues par les interviewés étaient classées en cinq catégories d'usage (Figure 2). S'agissant de la consommation des organes, seuls les fruits étaient consommés par les humains. La commercialisation concernait le bois de chauffe, les fruits, l'huile issue des graines et la plante parasite. Pour ce qui est des parties ou organes de la plante utilisés, tous les groupes ethnolinguistiques utilisaient presque les mêmes organes excepté l'huile issue des graines. 


\section{Caractéristiques des sous-variétés de $S$. guineense var. macrocarpum selon les Mboum}

A l'analyse, le groupe ethnolinguistique Mboum utilisaient quatre principaux critères pour distinguer les quatre sous-variétés de $S$. guineense var. macrocarpum (Tableau 2). Toutes ces sousvariétés étaient identiques de par la couleur des fruits non-mûrs (vert). A maturité complète, la différence entre celles-ci était visible à partir de la couleur des fruits. Par ailleurs, la présence des fissures y était remarquable. Cependant, la sous-variété 3 (gormagna) était particulière de par la constance de la couleur (vert) de ses fruits immatures et mâtures. Pour ce qui est de la couleur des endocarpes des graines, la sous-variété gormagna (blanchâtre) se distinguait des trois autres (violet). Concernant le goût des fruits mûrs, il variait de: amer (sous-variété lembali ngambgwar) à sucré (sous-variété gormagna) en passant par peu sucré (sous-variétés langaou 1 et langaou 2). A partir des caractéristiques paysannes Mboum, les sous-variétés langaou 1 (Figure 3a) et langaou 2 (Figure 3d) étaient quasi identiques. Seule la couleur de leurs fruits mûrs évitait la confusion. En général, les hommes $(71,59 \%)$ maitrisaient mieux la plante que les femmes $(28,41 \%)$. Quant à l'âge des interviewés, les adultes (75\%) avaient plus connaissance de la ressource végétale que les jeunes $(25 \%)$. Concernant des informations sur les sous-variétés, celle langaou 1 était la mieux connue $(51,14 \%)$. Cependant, la sous-variété lembali ngambgwar (Figure 3c) était la moins signalée $(3,40 \%)$. Au regard du genre et l'âge, très peu de femmes $(4,54 \%)$ et de jeunes $(2,27 \%)$ avaient connaissance de la sousvariété gormagna (Figure 3b).
Valeurs d'usage ethnobotanique total (VUET) des sous-variétés de Syzygium guineense var. macrocarpum

Les valeurs d'usage ethnobotanique total (VUET) des sous-variétés langaou 1 et langaou 2 ont été regroupées du fait de leur rapprochement (Tableau 2). Ce regroupement explique la présence de trois VUET au lieu de quatre (Tableau 3). Les VUET des sousvariétés variaient en fonction du genre et de l'âge. Pour ce qui est du genre, la sous-variété présentant une VUET $(4,51)$ élevée était gormagna selon les hommes. Cependant, les femmes affirmaient que la sous-variété langaou (1 et 2) avait la plus importante VUET $(0,75)$. Concernant l'âge, les jeunes trouvaient que la sous-variété langaou (1 et 2) possédait la meilleur VUET $(2,85)$. Par contre, chez les adultes, c'était la sous-variété gormagna $(4,28)$. Par rapport au genre ou à l'âge, la sous-variété lembali ngambgwar avait une VUET inférieure à celle des deux autres. Pour ce qui est du rang occupé par les sousvariétés, celle gormagna occupait le rang numéro 1 aussi bien chez les hommes que chez les adultes. Cependant, elle était classée au second plan chez les femmes et les jeunes. Par contre, la sous-variété langaou (1 et 2) était en pôle position chez les femmes et les jeunes. En outre, elle occupait la deuxième place chez les hommes et les adultes. A la différence des sous-variétés suscitées, lembali ngambgwar était classé troisième chez les hommes, les femmes, les jeunes et les adultes. 


\section{G. M. LAMY LAMY et al. / Int. J. Biol. Chem. Sci. 12(4): 1636-1649, 2018}

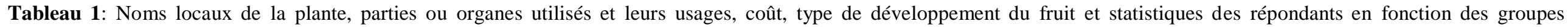
ethnolinguistiques des Hautes Savanes Guinéennes (Adamaoua, Cameroun).

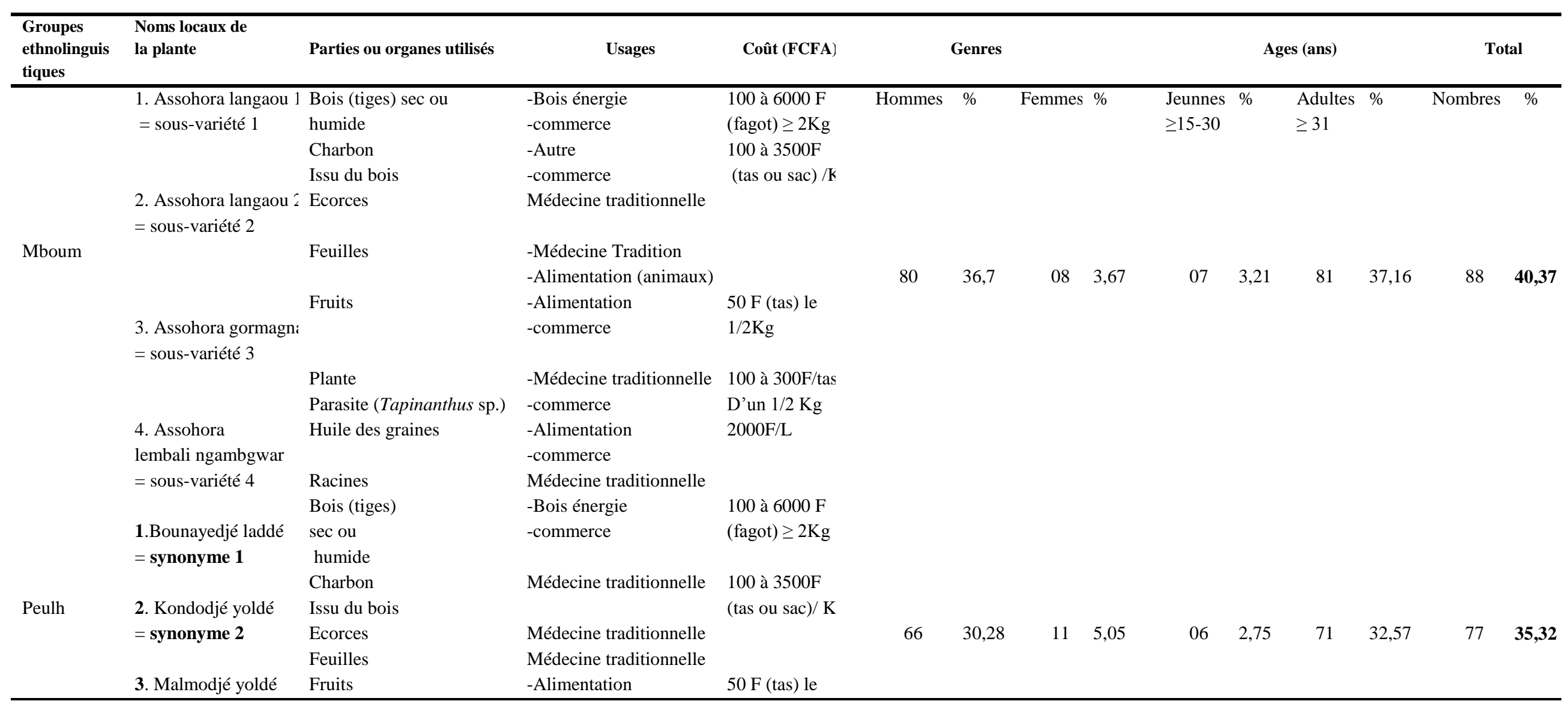




\begin{tabular}{|c|c|c|c|c|c|c|c|c|c|c|c|c|c|c|}
\hline & $=$ synonyme 3 & & -commerce & $1 / 2 \mathrm{Kg}$ & & & & & & & & & & \\
\hline & & Plante & -Médecine traditionnelle & 100 à $300 \mathrm{~F} / \mathrm{tas}$ & & & & & & & & & & \\
\hline & 4. Sikakondohi yoldé & Parasite (Tapinanthus sp.) & -commerce & D'un $1 / 2 \mathrm{Kg}$ & & & & & & & & & & \\
\hline & $=$ synonyme 4 & Huile & -Alimentation & & & & & & & & & & & \\
\hline & & des graines & -commerce & 2000F (Litre) & & & & & & & & & & \\
\hline & & Racines & Médecine traditionnelle & & & & & & & & & & & \\
\hline & & Bois (tiges) & -Bois énergie & 100 à $6000 \mathrm{~F}$ & & & & & & & & & & \\
\hline & & sec ou & -commerce & (fagot) $\geq 2 \mathrm{Kg}$ & & & & & & & & & & \\
\hline & & humide & & & & & & & & & & & & \\
\hline \multirow{3}{*}{ Gbaya } & \multirow{3}{*}{ Kélou } & Ecorces & Médecine traditionnelle & & & & & & & & & & & \\
\hline & & Feuilles & $\begin{array}{l}\text { Médecine traditionnelle } \\
\text {-Alimentation }\end{array}$ & & 18 & 08,26 & 01 & 0,46 & 03 & 01,38 & 16 & 07,34 & 19 & $\mathbf{0 8 , 7 2}$ \\
\hline & & Fruits & -commerce & $\begin{array}{l}50 \mathrm{~F} \text { (tas) le } \\
1 / 2 \mathrm{Kg}\end{array}$ & & & & & & & & & & \\
\hline \multirow{15}{*}{$\begin{array}{c}\text { Dii } \\
\text { (Dourou) }\end{array}$} & \multirow{15}{*}{ Assohora } & Plante & -Médecine traditionnelle & 100 à $300 \mathrm{~F} /$ tas & \multirow{15}{*}{10} & \multirow{15}{*}{0,46} & \multirow{15}{*}{02} & \multirow{15}{*}{0,92} & \multirow{15}{*}{04} & \multirow{15}{*}{01,83} & \multirow{15}{*}{08} & \multirow{15}{*}{03,67} & \multirow{15}{*}{12} & \multirow{15}{*}{$\mathbf{5 , 5 0}$} \\
\hline & & $\begin{array}{l}\text { Parasite } \\
\text { (Tapinanthus sp.) }\end{array}$ & -commerce & D'un $1 / 2 \mathrm{Kg}$ & & & & & & & & & & \\
\hline & & Racines & Médecine traditionnelle & & & & & & & & & & & \\
\hline & & Bois (tiges) & -Bois énergie & 100 à $6000 \mathrm{~F}$ & & & & & & & & & & \\
\hline & & $\mathrm{sec}$ ou & -commerce & (fagot) 5 à $50 \mathrm{k}$ & & & & & & & & & & \\
\hline & & humide & & & & & & & & & & & & \\
\hline & & Ecorces & Médecine traditionnelle & & & & & & & & & & & \\
\hline & & Feuilles & Médecine traditionnelle & & & & & & & & & & & \\
\hline & & Fruits & Alimentation & $50 \mathrm{~F}$ (tas) le & & & & & & & & & & \\
\hline & & & -commerce & $1 / 2 \mathrm{Kg}$ & & & & & & & & & & \\
\hline & & Plante & -Médecine traditionnelle & 100 à $300 \mathrm{~F} /$ tas & & & & & & & & & & \\
\hline & & Parasite (Tapinanthus sp.) & -commerce & D'un $1 / 2 \mathrm{Kg}$ & & & & & & & & & & \\
\hline & & Bois (tiges) & Bois énergie & 100 à $6000 \mathrm{~F}$ & & & & & & & & & & \\
\hline & & $\sec$ ou & -commerce & (fagot) $\geq 2 \mathrm{Kg}$ & & & & & & & & & & \\
\hline & & humide & & & & & & & & & & & & \\
\hline
\end{tabular}


G. M. LAMY LAMY et al. / Int. J. Biol. Chem. Sci. 12(4): 1636-1649, 2018

\begin{tabular}{|c|c|c|c|c|c|c|c|c|c|c|c|c|c|c|}
\hline \multirow[t]{8}{*}{ Laka } & \multirow[t]{8}{*}{ Assohora } & Ecorces & \multicolumn{12}{|l|}{ Médecine traditionnelle } \\
\hline & & Feuilles & Médecine traditionnelle & & 17 & 07,8 & 01 & 0,46 & 00 & 00 & 18 & 08,26 & 18 & 08,26 \\
\hline & & Fruits & -Alimentation & $50 \mathrm{~F}$ (tas) le & & & & & & & & & & \\
\hline & & & -commerce & $1 / 2 \mathrm{Kg}$ & & & & & & & & & & \\
\hline & & Plante & -Médecine traditionnelle & 100 à $300 \mathrm{~F} / \mathrm{tas}$ & & & & & & & & & & \\
\hline & & Parasite (Tapinanthus sp.) & -commerce & D'un $1 / 2 \mathrm{Kg}$ & & & & & & & & & & \\
\hline & & Bois (tiges) & -Bois énergie & 100 à $6000 \mathrm{~F}$ & & & & & & & & & & \\
\hline & & $\mathrm{sec}$ ou & -commerce & $($ fagot $) \geq 2 \mathrm{Kg}$ & & & & & & & & & & \\
\hline \multirow[t]{8}{*}{ Ngambaye } & Assohora & humide & & & 04 & 01,83 & 00 & 00 & 00 & 00 & 04 & 01,83 & 04 & 01,83 \\
\hline & & Ecorces & Médecine traditionnelle & & & & & & & & & & & \\
\hline & & Feuilles & Médecine traditionnelle & & & & & & & & & & & \\
\hline & & Fruits & -Alimentation & $50 \mathrm{~F}(\operatorname{tas}) \mathrm{le}$ & & & & & & & & & & \\
\hline & & & -commerce & $1 / 2 \mathrm{Kg}$ & & & & & & & & & & \\
\hline & & Plante & -Médecine traditionnelle & 100 à $300 \mathrm{~F} / \mathrm{tas}$ & & & & & & & & & & \\
\hline & & Parasite (Tapinanthus sp.) & -commerce & D'un $1 / 2 \mathrm{Kg}$ & & & & & & & & & & \\
\hline & & Total & & & 195 & 89,45 & 23 & 10,55 & 20 & 09,17 & 198 & 90,83 & 218 & 100 \\
\hline
\end{tabular}


G. M. LAMY LAMY et al. / Int. J. Biol. Chem. Sci. 12(4): 1636-1649, 2018

Tableau 2 : Description des sous-variétés de Syzygium guineense var. macrocarpum selon le groupe ethnolinguistique Mboum dans les Hautes Savanes Guinéennes

\begin{tabular}{|c|c|c|c|c|c|c|c|c|c|c|c|c|c|c|c|c|}
\hline \multirow[b]{2}{*}{$\begin{array}{l}\text { Groupe } \\
\text { ethnolinguistique }\end{array}$} & \multirow[b]{2}{*}{$\begin{array}{l}\text { Noms des } \\
\text { Morphotype }\end{array}$} & \multirow[b]{2}{*}{$\begin{array}{l}\text { Noms des } \\
\text { Sous-variétés } \\
\text { en Mboum }\end{array}$} & \multirow[b]{2}{*}{$\begin{array}{l}\text { Couleurs des } \\
\text { fruits non mûrs }\end{array}$} & \multicolumn{3}{|c|}{ Caractéristiques Paysannes des fruits } & \multicolumn{4}{|c|}{ Genres } & \multicolumn{3}{|c|}{ Ages (ans) } & \multicolumn{3}{|c|}{ Total } \\
\hline & & & & $\begin{array}{l}\text { Couleurs des } \\
\text { fruits Mûrs }\end{array}$ & $\begin{array}{l}\text { Couleurs } \\
\text { Extérieures } \\
\text { des endocarpes }\end{array}$ & $\begin{array}{l}\text { Goûts } \\
\text { des } \\
\text { Fruits mûrs }\end{array}$ & Hommes & $\%$ & Femmes & s \% & $\begin{array}{l}\text { Jeunes } \\
\geq 15-30\end{array}$ & $\%$ & $\begin{array}{l}\text { Adulte } \\
\geq \mathbf{3 1}\end{array}$ & $\%$ & Nombres & $\%$ \\
\hline \multirow{6}{*}{ Mboum } & Sous-variété & $\begin{array}{l}\text { Assohora } \\
\text { langaou } 1\end{array}$ & Vert & $\begin{array}{l}\text { Noir/violet-foncé } \\
\text { (fissures) }\end{array}$ & violet & Peu sucré & 30 & 34,1 & 15 & 17,04 & 13 & 13 & 32 & 36,36 & 45 & $\mathbf{5 1 , 1 4}$ \\
\hline & & Assohora & & Violet-vert (fissures) & & & & & & & & & & & & \\
\hline & Sous-variété & Langaou 2 & Vert & & violet & Peu sucré & 11 & 12,5 & 06 & 6,82 & 07 & 07 & 10 & 11,36 & 17 & 19,32 \\
\hline & Sous-variété & $\begin{array}{l}\text { Assohora } \\
\text { gormagna }\end{array}$ & Vert & Vert (fissures) & Blanchâtre & sucré & 19 & 21,59 & 04 & 4,54 & 02 & $\begin{array}{l}02 \\
7\end{array}$ & 21 & 23,86 & 23 & 26,14 \\
\hline & Sous-variété & $\begin{array}{l}\text { Assohora } \\
\text { Lembali } \\
\text { ngambgwar }\end{array}$ & Vert & Violet (fissures) & violet & Amer & 03 & 3,41 & 00 & 00 & 00 & 00 & 03 & 3,41 & 03 & 3,40 \\
\hline & & & Total & & & & 63 & 71,59 & 252 & 28,41 & 22 & 25 & 566 & 75 & 88 & 100 \\
\hline
\end{tabular}

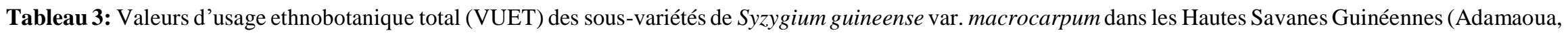
Cameroun)

\begin{tabular}{llccccccc}
\hline \multicolumn{1}{c}{ Sous-variétés } & \multicolumn{3}{c}{ Genres } & \multicolumn{2}{c}{ Ages (ans) } \\
\cline { 2 - 7 } & VUET & Hommes & RANG & VUET & RANG & VUE & RANG & VUET \\
Langaou (1et 2) & 4,26 & $\mathbf{2}$ & $\mathbf{0 , 7 5}$ & $\mathbf{1}$ & $\mathbf{2 , 8 5}$ & $\mathbf{1}$ & 3,58 \\
Gormagna & $\mathbf{4 , 5 1}$ & $\mathbf{1}$ & 0,49 & $\mathbf{2}$ & 0,75 & $\mathbf{2}$ & $\mathbf{4 , 2 8}$ & $\mathbf{2}$ \\
Lembali ngambgwa & 01 & $\mathbf{3}$ & 00 & $\mathbf{3}$ & 00 & $\mathbf{1}$ & 01 \\
\hline
\end{tabular}




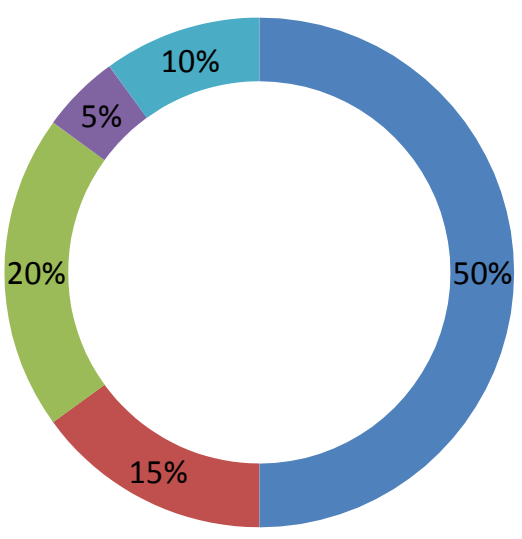

alimentation

bois énergie

médecine

traditionnelle

commerce

autres

Figure 2 : Principaux usages de $S$. guineense var. macrocarpum dans les Hautes Savanes Guinéennes (Adamaoua, Cameroun).
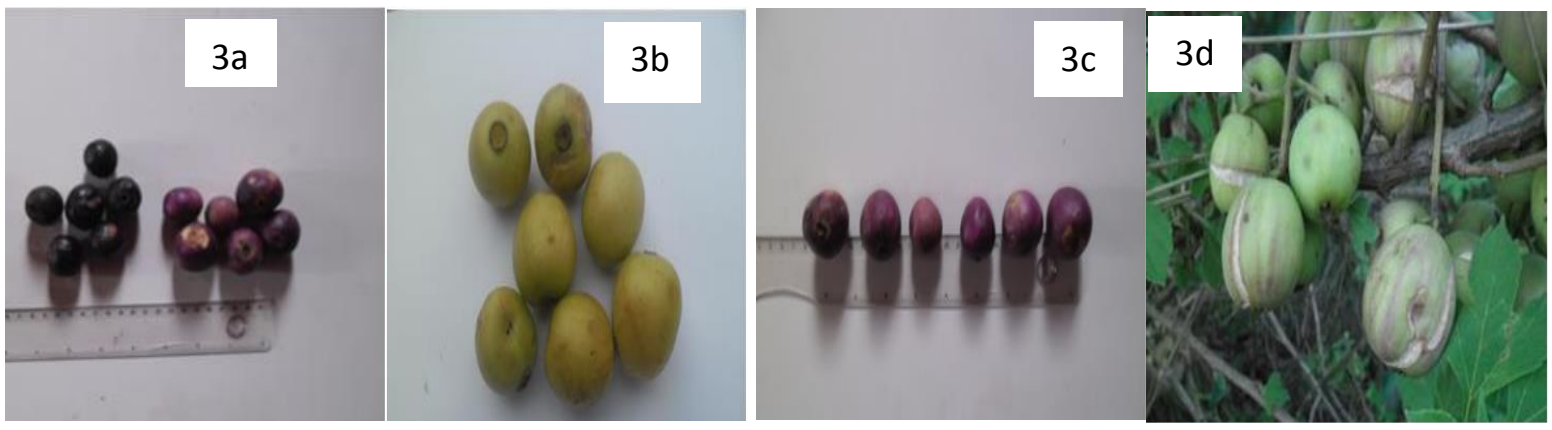

Figure 3: Images photographiques des fruits des sous-variétés de Syzygium guineense var. macrocarpum dans les Hautes Savanes Guinéennes (Adamaoua, Cameroun). 3a : sous-variété langaou 1; 3b : sous-variété gormagna; 3c: sous-variété lembali ngambgwar; 3d: sous-variété langaou 2. (Photo. LAMY).

\section{DISCUSSION}

Savoirs endogènes et noms locaux de $S$. guineense var. macrocarpum dans les Hautes Savanes Guinéennes

L'étude ethnobotanique révèle qu'il existe un savoir local sur Syzygium guineense var. macrocarpum dans les Hautes Savanes Guinéennes (Adamaoua, Cameroun). Pareille étude avait permis de documenter les valeurs d'usage et les connaissances traditionnelles de nombreuses autres plantes en Afrique de l'Ouest (Akabassi et al., 2017 ; Yedomonhan et al., 2017). Dans la zone agro-écologique des Hautes Savanes Guinéennes, les adultes (75\%) avaient plus de savoirs sur la ressource végétale que les jeunes $(25 \%)$ et les Hommes $(71,59 \%)$ maîtrisaient mieux la plante que les Femmes $(28,41 \%)$. Ces résultats sont accord avec ceux rapportés par Kouyaté et al. (2015). En effet, selon ces auteurs, l'âge et le sexe présentent un intérêt capital car ils impactent sur les stratégies locales de gestion des ressources naturelles. En ce qui concerne les noms locaux attribués à la plante, elle peut être désignée soit par un nom local (Assohora), soit par quatre synonymes ou par quatre noms différents dont chacun représente une sous-variété. Ces résultats sont similaires à ceux trouvés 
précédemment sur un nom et la synonymie nominale de la plante (Ambé, 2001; Mapongmetsem et al., 2012). Pour ce qui est des sous-variétés, le respect des règles de nomenclature botanique recommande, l'ajout du préfixe «sous » au taxon concerné lorsque ce dernier révèle des taxons inférieurs (Greuter et al., 2000; Piroux, 2002). Bien que l'existence des sous-variétés de la plante ait été rapportée (Zigelski, 2016), le chiffre ou le nombre exacte de ces dernières n'avait pas encore été mentionnée. Le manque de valeur chiffrée des sous-variétés de la plante pourrait s'expliquer par le fait que, la plante n'intéresse pas encore véritablement la communauté internationale malgré ses multiples avantages et sa disparition progressive. Selon Ouattara et al. (2016), le statut de la plante n'a pas encore véritablement été pris en compte dans la liste rouge des plantes menacées de l'Union Internationale pour la Conservation de la Nature (UICN). Les différentes désignations de la plante basées sur les caractéristiques des fruits traduisent le fait que, Syzygium guineense var. macrocarpum présente une variabilité morphologique dans les Hautes Savanes Guinéennes. Ces résultats sont similaires à ceux précédemment rapportés sur les fruits d'autres espèces végétales (Abdoulaye et al., 2016 ; Wallis et al., 2016).

\section{Parties et organes de la plante utilisés et leurs utilisations dans les Hautes Savanes Guinéennes}

Tous les organes (écorce, feuilles, fruits et graines) de la plante sont utilisés par l'ensemble des groupes ethnolinguistiques interrogés. Ces résultats sont en accord avec ceux trouvés par Arbonnier (2009). Cet intérêt pour Syzygium guineense var. macrocarpum traduit une pression anthropique sur la ressource visant à la faire disparaître ex situ. L'huile issue des graines des fruits de la plante est un produit dérivé de valeur ajouté. Cependant, elle n'a pas encore véritablement été prise en compte dans les travaux de recherches. Ceci pourrait s'expliquer par le fait que cette huile ne soit pas encore vulgarisée. Ainsi, une valeur ajoutée sera apportée à cette substance oléagineuse dont le prix est largement supérieur à celui de nombreuses huiles commercialisées dans les marchés. Pour ce qui est du fruit, celui de la sous-variété gormagna présentait une particularité en demeurant vert mûr et non mûr. Ceci pourrait s'expliquer par la physiologie de maturation spéciale de cette sous-variété. S'agissant de la consommation, l'unique organe (partie) de la plante consommé dans les Hautes Savanes Guinéennes était le fruit. La consommation du fruit de $S$. guineense var. macrocarpum avait déjà été rapportée (Loura et al., 2000 ; Alexandre, 2002; Mapongmetsem, et al., 2009; Mapongmetsem et al., 2012). Cette consommation du fruit par tous les groupes ethnolinguistiques ayant participés à l'étude peut se traduire par le fait que ce dernier contribue à l'équilibre alimentaire dans la zone d'étude. Les fruits de Syzygium guineense var. macrocarpum sont une solution aux problèmes d'insécurité alimentaire dont les populations des Hautes Savanes Guinéennes sont victimes (FAO, 2015).

\section{Calcul des valeurs d'usage ethnobotanique total (VUET) des sous-variétés du végétal}

Le calcul des valeurs d'usage ethnobotanique total (VUET) des sous-variétés de Syzygium guineense var. macrocarpum a permis leur évaluation. Les sous-variétés gormagna (VUET $=4,51$ ) et langaou 1 et 2 (VUET $=4,26)$ étaient les plus prisés selon les hommes. Chez les adultes, la même tendance était observée pour les sous-variétés gormagna $($ VUET $=4,28)$ et langaou 1 et 2 (VUET = $3,58)$. Ces résultats corroborent ceux de nombreux auteurs ayant rapporté une VUET $\geq$ 3 attribuée aux espèces prisées (rangs élevés) par les riverains (Belem et al., 2008 ; Dossou et al., 2012). En effet, Belem et al. (2008) ont trouvé que les espèces préférées dans les villages Dem et Wèdsè au Burkina Faso avaient une valeur d'usage ethnobotanique supérieure à 3. Par ailleurs, Dossou et al. (2012) trouvaient que la valeur d'usage ethnobotanique de l'espèce la plus exploitée et prisée (Diallium guineense) était égale à 8,98. Cette différence entre les valeurs d'usage ethnobotanique des études et largement supérieur à celle seuil (Belem et al., 2008) 
pourrait s'expliquer par le fait qu'elle varie en fonction du genre, de l'âge des répondants et du lieu'étude. Au contraire des précédents interviewés, les femmes (VUET $=0,75)$ et les jeunes (VUET $=2,85)$ déclaraient que c'était plutôt les sous-variétés langaou 1et 2 qui étaient les plus prisées pour l'ensemble des catégories. Tandis que les sous-variétés gormagna $\quad(\mathrm{VUET}=0,49)$ et lembali ngambgwar (VUET=01) de par leur rang étaient les moins appréciés (Tableau 3). La baisse de ces valeurs d'usages ethnobotaniques par rapport à celle seuil $($ VUET $=3$ ) pourrait s'expliquer par le fait que c'étaient les femmes et les enfants qui récoltaient et commercialisaient les PFNL de la plante. Cependant, les femmes étaient interdites de parler aux étrangers ou de sortir en journée dans certaines localités où s'est effectuée l'étude. Elles affirmaient parcourir plusieurs kilomètres dans la savane arborée pour espérer trouver un pied de la sous-variété gormagna qui serait en voie de disparition. En outre, seuls les jeunes ayant vécu en campagne et les jeunes bergers avaient connaissance de l'existence des sous-variétés de Syzygium guineense var. macrocarpum.

\section{Conclusion}

L'étude ethnobotanique a révélé que l'espèce Syzygium guineense var macrocarpum était utilisée par les populations des Hautes Savanes Guinéennes. Le fruit a été la partie la plus prisée et l'alimentation l'usage fréquent. Quatre sous-variétés ayant des noms distincts ont été décrites. La description de ces sous-variétés dépend des caractéristiques du fruit. L'évaluation des sous-variétés a été fonction de l'âge, du genre et du groupe ethnolinguistique. Les sous-variétés 1, 2 et 3 avaient des valeurs d'usages ethnobotaniques les plus importantes. L'utilisation incontrôlée de toutes les parties (organes) de la plante appelle à des mesures de conservation de la ressource dans les systèmes de production paysans et à sa préservation dans des zones protégées. Les techniques de domestication appropriées à Syzygium guineense var. macrocarpum, de faibles coût et accessibles aux riverains sont indispensables. Pour une conservation durable de la plante, des études de variabilité morphologique et moléculaires de ses sous-variétés permettront la sélection de celles ayant des valeurs significatives. Des études de caractérisation de l'huile issue des graines des fruits de la plante permettront de prévenir des éventuels cas de toxicités de celleci. Des recherches en génomique et en physiologie de maturation devraient être menées pour comprendre la particularité de la sous-variété 3 (gormagna).

\section{CONFLIT D'INTERETS}

Les auteurs déclarent qu'il n'y a pas de conflit d'intérêt.

\section{CONTRIBUTIONS DES AUTEURS}

PMM a initié l'étude, supervisé les enquêtes de terrain et relu le manuscrit; AI a traité les données et fourni la documentation ; DN a contribué à la conception du protocole et fourni la documentation; GMLL a mené les enquêtes de terrain et contribué à la rédaction du manuscrit.

\section{REMERCIEMENTS}

Les auteurs remercient les populations de la zone d'étude pour l'hospitalité et le partage des savoirs endogènes. Ils remercient également les lecteurs anonymes pour leur contribution scientifique ayant permis l'amélioration de cet article.

\section{REFERENCES}

Abdoulaye B, Béchir AB, Mapongmetsem PM. 2016. Variabilité morphologique de Balanites aegyptiaca (L.) Del. dans la région du Ouaddaï au Tchad. Int. J. Biol. Chem. Sci., 10(4) : 1733-1746. DOI : http://dx.doi.org/10.4314/ijbcs.v10i4.23.

Akabassi GC, Padonou EA, Chadare FJ, Assogbadjo AE. 2017. Importance ethnobotanique et valeur d'usage de Picralima nitida (stapf) au Sud-Bénin (Afrique de l'Ouest). Int. J. Biol. Chem. Sci., 11(5): 1979-1993. DOI : https://dx.doi.org./10.4314/ijbcs.v11i5.4.

Alexandre DY. 2002. Initiation à l'Agroforesterie en Zone Sahélienne: les Arbres des Champs du Plateau 
Central du Burkina-Faso. Ird-Kartala : Paris.

Ambé ASA, Ouattara D, Tiébré MS, Vroh BTA, Zirihi GN, N'guessan EK. 2015. Diversité des plantes médicinales utilisées dans le traitement traditionnel de la diarrhée sur les marchés d'Abidjan (Côte d'Ivoire). Journal of Animal \& Plant Sciences, 26: 4081-4096. DOI: http://www.m.elewa.org/JAPS

Ambe GA. 2001. Les fruits sauvages comestibles des savanes guinéennes de Côte-d'Ivoire : état de la connaissance par une population locale, les Malinké. Biotechnol. Agron. Soc. Environ., 5(1): 43-58.

Arbonnier M. 2009. Arbres, arbustes et lianes des zones sèches d'Afrique de l'Ouest. Quæ: Versailles.

Belem B, Smith OC, Théilade I, Bellefontaine R, Guinko S, Lykke AM, Diallo A, Boussim JI. 2008. Identification des arbres hors forêts préférés des populations du Sanmatenga (Burkina Faso). Bois et Forêts des Tropiques, 298 (4) : 53-64

Boulvert Y. 1986. République Centrafricaine, carte phytogéographioue à $1 / 1.000 .000$ (feuilles Est et Ouest). ORSTOM : Paris.

Deffo V, Ottou JF, Ombionyo M, Achundoh LE, Djoumessi M. 2009. Facteurs socioéconomiques affectant l'utilisation des sous-produits agro-industries pour l'embouche bovine à contre saison dans l'Adamaoua, Cameroun. Biotechnol. Agron. Soc. Environ., 13(3): 357-365.

Doumara GD, Mapongmetsem PM. 2013. Vegetative propagation of Syzygium guineense var. macrocarpum Engl. (Myrtaceae) by stem cutting. $20^{\text {th }}$ Annual Conference of the Cameroon Biosciences Society, 5-8 December 2013. Ngaoundere, Cameroon.

Dossou ME, Houessou GL, Lougbégnon OT, Tenté AHB, Codjia JTC. 2012. Etude ethnobotanique des ressources forestières ligneuse de la forêt marécageuse d'Agonvè et terroirs connexes au Bénin. Tropicultura, $\quad$ 30(1): 41-48. https://www.researchgate.net/publication 1259558319 .
FAO. 2015. Regional overview of food insecurity: African food security prospects brighter than ever. FAO: Accra, Ghana.

Greuter W, Mcneill J, Barrie FR, Burdet HM, Demoulin V, Filgueiras TS, Nicolson DH, Skog JE, Silva PC, Trehane P, Turland NJ, Hawksworth DL. 2000. Code international de nomenclature botanique de St Louis, trad. Malécot V. \& Soca R. 1ère éd. Koeltz Scientific: Germany.

Ibrahima A, Biyanzi P, Halima M. 2008. Changes in organic compounds during leaf litter leaching: laboratory experiment on eight plant species of the Sudanoguinea Savannas of Ngaoundere, Cameroon. iForest, 1: 27-33. http://www.sisef.it/iforest/.

Jauzein P. 1998. Opinion sur l'espèce végétale, sa taxinomie et sa nomenclature. Sauve qui peut, 10: 1-22.

Kimpouni V. 2009. Contribution à l'inventaire et à l'analyse de la flore ligneuse du plateau des Cataractes (CongoBrazzaville). Acta Botanica Gallica, 156: 233-244

Kouyaté AM, Dembélé U, Lykké AM. 2015. Les espèces ligneuses locales à huile: une ressource utile pour les communautés locales au Sud du Mali. Int. J. Biol. Chem. Sci., 9(6): 2754-2763. DOI: http://dx.doi.org/10.4314/ijbcs.v9i6.19.

Lougbegnon BAH, Tente M, Amontcha M, Codjia JTC. 2011. Impact culturelles et valeur d'usage des ressources végétales de la réserve forestière marécageuse de la vallée de Satatunga et zones connexes. Bulletin de la Recherche Agronomique du Bénin, 70: 11.

Loura BB, Mapongmetsem PM, Nkongmeneck BA, Foko J, Kapchie V, Denedoumba D. 2000. Caractérisation physico-chimique de deux Myrtacées endémiques des savanes soudano-guinéennes. Cam. J. Biol. Bioch. Sci., 10: 61-69.

Mapongmetsem PM, Hamawa Y, Niwah CB, Froumsia M, Zigro L, Meiga OS. 2009. Conservation et valorisation de la biodiversité dans les agrofôrets de case de la zone Soudano-Guinéenne. In Systematics and conservation of African 
Plants; Burgt XVD, Maesen JVD, Onana J-M (eds). Royal Botanic Gardens: Kew; 375-384.

Mapongmetsem PM, Kapchie VN, Tefempa BH. 2012. Diversity of local fruit trees and their contribution in sustaining the rural livelihood in the northern Cameroon. Ethiopian Journal of Environmental Studies and Management, 5: $32-46$.

Mapongmetsem PM, Laïssou M. 2010. Contribution à la domestication des fruitiers indigènes des savanes soudanoguinéennes: Influence du substrat et des substances de croissances sur l'enracinement des marcottes. International symposium on indigenous fruit trees for dryland Africa: Domestication for use in a changing environment, Centre CIEVRA Allada (Republic of Benin) 25-27 octobre 2010.

Maroyi A. 2008. Syzygium guineense (Willd.) DC. In: Louppe, D., Oteng-Amoako, A.A. \& Brink, M. (Editors). PROTA (Plant Resources of Tropical Africa / Ressources végétales de l'Afrique tropicale), Wageningen, Netherlands. (20/02/2018). http://uses.plantnetproject.org/fr/Syzygium_guineense_(PR OTA).

Nikema A, Ouedraogo SJ, Boussim J. 2001. Situation des ressources génétiques forestières du Burkina Faso. Atelier sousrégional FAO/IPGRI/ICRAF sur la conservation, la gestion, l'utilisation durable et la mise en valeur des ressources génétiques forestières de la zone sahélienne. Note thématique sur les ressources génétiques forestières, document FGR/22F, Ouagadougou, 2224 septembre, 1998. Département des forêts, FAO : Rome, Italie.

Ouattara D, Kouame D, Tiebre M.S, Yao JC, Kouadio, N'guessan KE. 2016. Biodiversité végétale et valeur d'usage en zone soudanienne de la Côte d'Ivoire. Int. J. Biol. Chem. Sci., 10: 1122-1138.

Piroux A. 2002. Evolution des Classifications Botaniques: Utilitaires, Morphologiques, Phylogéniques. ESSIB : Lyon.

Tankeu FN, Pieme CA, Nya CPB, Njimou RJ, Moukette BM, Chianese A, Ngogang JY.
2016. In vitro organo-protective effect of bark extracts from Syzygium guineense var macrocarpum against ferric nitrilotriacetateinduced stress in wistar rats homogenates. BMC Complementary and Alternative Medicine, 16: 315. DOI: 10.1186/s12906-016-1263-1.

Tchobsala, Mbolo, M., 2013. Characterization and impact of wood logging on plant formations in Ngaoundéré District, Adamawa Region, Cameroon. Journal of Ecology and the Natural Environment, 5: 265-277. DOI: 10.5897/JENE10.102.

Tchuenguem FFN. 2005. Foraging and pollination activity of Apis mellifera adansonii Latreille (Hymenoptera: Apidae, Apinae) onflower of three plants at Ngaoundéré (Cameroon): Callistemon rigidus (Myrtaceae), Sygygium guineense var. macrocarpum (Myrtaceae) and Voacanga Africana (Apocynaceae). State doctorate thesis, University of Yaoundé I, Yaoundé (Cameroon), p. 103.

Wallis ZN, Bagnan MA, Akossou AYJ, Kanlindogbe CB. 2016. Caractérisation morphologique d'une collection de fruits d'anacardier provenant de la commune de Parakou (Bénin). Int. J. Biol. Chem. Sci., 10(6): 2413-2422. http://ajol.info/index.php/ijbcs.

WCSP. 2018. World Checklist of Selected Plant Families. www.theplantlist.org (20/02/2018).

Yedomonhan H, Adomou AC, Aguessy M, Bossou FG. 2017. Evaluation des caractéristiques ethnobotaniques et structurales de Nesogordinia kabingaensis (K. Schum.) Capuron ex R. Germ. (Sterculiaceae) dans la forêt sacrée d'Ewè au Bénin en vue de la definition des strategies de sa conservation. Int. J. Biol. Chem. Sci., 11 (5): 2481-2494. DOI: https://dx.doi.org./10.4314/ijbcs.v11i5.4 2.

Zigelski P. 2016. Molecular biogeography of the Syzygium guineense complex: How environment and genetics push suffrutication in Africas Miombo region. Master Thesis, Universität Hamburg, p. 109. 